\title{
VARIACIONES Y CARACTERÍSTICAS EN LOS CAMBIOS DE NIVEL DE LA LAGUNA CRATÉRICA DEL VOLCAN IRAZÚ (1965-2012), COSTA RICA
}

\section{VARIATIONS AND CHARACTERISTICS IN CHANGES OF THE CRATER LAKE LEVEL OF IRAZÚ VOLCANO (1965-2012), COSTA RICA}

\author{
Ronald Ramírez $^{1,4 *}$, Cecilia Cordero ${ }^{2} \&$ Guillermo E. Alvarado ${ }^{3}$ \\ ${ }^{1}$ C.S. Recursos Geotérmicos, Instituto Costarricense de Electricidad, \\ Apdo. 10032-1000, Costa Rica \\ ${ }^{2}$ Servicio Unificado, Instituto Costarricense de Electricidad, \\ Apdo 10032-1000, Costa Rica \\ ${ }^{3}$ Área de Amenazas y Auscultación Sísmica y Volcánica, Instituto \\ Costarricense de Electricidad, Apdo 10032-1000, Costa Rica \\ ${ }^{4}$ Grupo Espeleológico Anthros. P.O Box 381-2070. Sabanilla, Costa Rica \\ *Autor para contacto: rgeoramirez@gmail.com
}

(Recibido: 25/2/2013/; aceptado: 11/6/2012)

\begin{abstract}
The small and ephemeral crater lake of the Irazú has presented noticeable changes in its coloring, temperature, $\mathrm{pH}$, and depth from 1965 until 2012. The level has varied from $31 \mathrm{~m}$ in 1995 and 2001 to stay completely dry in 1977, 1979, 1982-83, 1987 and 1990. These levels are not always directly related to the amount of rainfall. It seems that at times the waterproofed base of the lake was cracked allowing the lake to drain relatively quickly, leaving the base visible for several months or years, and after a time, formed again. The variation of the color (green, turquoise-blue, yellow, rare whitish and reddish), temperature $\left(16^{\circ}-35^{\circ} \mathrm{C}\right)$, and $\mathrm{pH}(2.85-5.85)$ seems to be related to the depth, underwater fumarolic activity, algae activity and ferruginous sediments produced by the constant landslides of the walls. Keywords: Crater Lake, waterproof bottom, coloration, seismicity, crater deepness, Irazú volcano, Costa Rica.
\end{abstract}

RESUMEN: La laguna cratérica del Irazú ha presentado cambios notorios en su coloración, temperatura, grado de acidez y profundidad desde 1965 hasta 2012. El nivel ha variado desde los $31 \mathrm{~m}$ en 1995 y 2001 hasta quedarse totalmente seca como sucedió principalmente en 1977, 1979, 1982-83, 1987 y 1990. Estos niveles no siempre se encuentran relacionados en forma directa con el grado de precipitación pluvial. En ciertos momentos, al parecer, en el fondo impermeabilizado de la laguna, se forman grietas que permiten drenar relativamente rápido, dejando el fondo visible por 
varios meses o años, para que tiempo después se vuelva a formar debido a una nueva selladura de las mismas. La variación del color (verde, turquesa azulado a amarillo, más raramente blanquecino y rojizo en las periferias), temperatura $\left(16^{\circ}-35^{\circ} \mathrm{C}\right), \mathrm{pH}(2,85-5,85)$ parece estar relacionada con la profundidad, actividad de fumarolas subacuáticas, actividad de algas y a la formación de sedimentos ferruginosos producto de los constantes deslizamientos de las paredes.

Palabras clave: Laguna cratérica, fondo impermeabilizado, coloración, sismicidad, profundidad laguna, volcán Irazú, Costa Rica.

\section{INTRODUCCIÓN}

El Parque Nacional Volcán Irazú (3432 m s.n.m), ubicado en la provincia de Cartago, posee varios atractivos turísticos, entre ellos, el paisaje semi-desértico de páramo volcánico, así como sus cráteres (Fig. 1). Este edificio volcánico se ha mantenido en constante vigilancia volcánica por instituciones encargadas como el OVSICORI y la Red Sismológica Nacional (RSN). El monitoreo a través del tiempo ha permitido obtener un registro sobre la intensidad de la actividad hasta la actualidad. Particularmente, el cráter Principal, resulta impresionante al visitante por su profundidad y bordes escarpados, al igual que por la presencia de la laguna cratérica intermitente (Alvarado, 2000).

Las variaciones en la coloración y la profundidad de la laguna del cráter principal del volcán Irazú son aspectos importantes, ya que son producto de cambios, que pueden tomarse como indicadores de futura actividad o como elementos de monitoreo y estudio. Esta laguna tiene la particularidad de que puede persistir por varios años, pero en un lapso de semanas se puede secar, mientras que un tiempo después, se vuelve a instaurar. Los cambios de color varían desde verde turquesa hasta blancuzco, pasando por tonos amarillentos y rojos y de temperaturas desde frías hasta templadas.

Quizás una de las primeras menciones sobre la particularidad del color de la laguna cratérica del Irazú, corresponde al afamado vulcanólogo francés, Tazieff (1972), quién comenta que el contenido de la laguna cratérica del Irazú, antes de su prolongada actividad de 1963 - 1965, era de color sangre de buey, posiblemente por las sales férricas.
Alvarado (1989) hipotetiza que la laguna cratérica del Irazú se puede secar, posiblemente debido a la infiltración de sus aguas por algunas grietas y por las bajas precipitaciones. Posteriormente, realiza un recuento general de la formación y desaparición de la laguna cratérica y sus colores asociados (Alvarado, 2000).

En el informe realizado por Van der Laat (2009), a razón de estudiar los procesos que ocurren en el volcán sobre el descenso del nivel de la laguna cratérica de 2003 a 2009, indica que las variaciones del nivel se originan por 3 posibles causas: a) el aporte de precipitación pluvial que se acumula en el interior del cráter, b) la infiltración y c) por efectos de compresión - distensión tectónica local que permite o impide la acumulación o infiltración de las aguas. Indica, además, que el cambio de coloración se debe a: i) derrumbes de paredes internas, ii) nivel de dilución de los sedimentos y iii) contenido mineral como azufre, algas, etc. Sin embargo, no aporta detalles, justificaciones ni analiza la información recopilada.

Dentro de los estudios geomorfológicos, Tassi et al. (2009) realizan en febrero del 2001, la batimetría de la laguna del Irazú utilizando un eco-sonar portable (Humminbird Legend 1005) y el software de geospacial Surfer 6.0, para producir las imágenes morfológicas. Ellos determinaron que la forma del lago es oblonga, con un diámetro promedio de entre 350 y $150 \mathrm{~m}$, una máxima profundidad de $31 \mathrm{~m}$ y un volumen de entre $5,1 \times 105$ y $3,7 \times 10^{5} \mathrm{~m}^{3}$.

Los registros de OVSICORI indican que la profundidad promedio de la laguna fue de $17,4 \mathrm{~m}$ para el mes de octubre del año 2001. Sin embargo, las contribuciones de los estudios de Tassi et al. (2009) indican una profundidad máxima de 31 


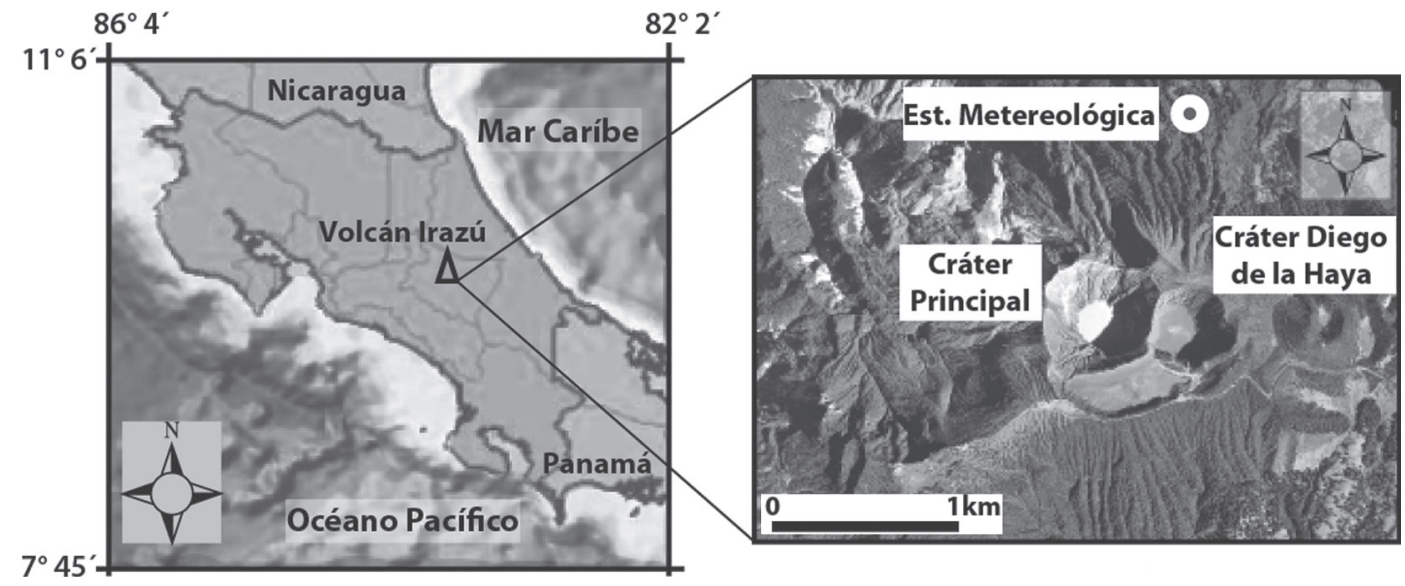

Fig. 1: Ubicación del cráter Principal del volcán Irazú.

m para ese mismo año, debido que el fondo de la laguna para ese entonces poseía una irregularidad (Fig. 2). Por lo tanto, en el momento en que se indica una profundidad de $17,4 \mathrm{~m}$ corresponde con la zona de topografía casi plana, mientras que los $31 \mathrm{~m}$ indican la profundidad en la zona de máxima depresión de la laguna.

El objetivo del presente trabajo es analizar con detalle la cronología y las razones posibles por las cuales ocurren estos eventos, con el fin de suministrar datos que permitan transmitir información y explicaciones lógicas de cómo y por qué se dan estas variaciones en la laguna.

\section{METODOLOGÍA}

Con el fin de analizar los datos del volcán Irazú, se compiló la documentación correspondiente a la coloración, los niveles del lago y los deslizamientos dentro del cráter desde 1965 al 2012. Dichos datos son el resultado del procesamiento de fotografías, periódicos, publicaciones, e informes varios sobre el estado del volcán. Además, se realizó la recolección de muestras de material del fondo de la laguna, fotografías tomadas durante el descenso tanto de la laguna, de las paredes del cráter como de la caverna ubicada en el flanco NW del volcán. Aparte de las publicaciones y reportes internos, se consultaron las páginas webs del OVSICORI (www.ovsicori.una. ac.cr/) y de la RSN (www.rsn.ucr.ac.cr) sobre el estado de los volcanes desde 1979 al 2012.

Un parámetro importante para el análisis de la dinámica de la laguna cratérica es la variación de su nivel. Para dicho análisis, se recopilaron datos sobre las variaciones relativas y absolutas del nivel del lago en diferentes años y se buscaron puntos de referencia dentro de las fotografías como las morfologías poco o nada cambiantes dentro del cráter, así como irregularidades de las paredes y mediciones directas cada $2 \mathrm{~m}$, aproximadamente, las cuales funcionan para uniformizar los niveles.

Se toma como referencia el nivel indicado en la Fig. 3 que corresponde al año 2011 (1,5 m), y a partir de este punto se empezaron a realizar las mediciones directas y su correspondiente comparación con las fotografías. Posteriormente, se realizó una correlación y homogenización entre los diferentes datos para obtener la profundidad promedio en metros desde 1965 al 2011 (Fig. 3).

Por otro lado, uno de los parámetros que se analiza, corresponde a la precipitación $(\mathrm{mm})$, para la cual se contó con la información del Instituto Meteorológico Nacional (IMN) sobre el promedio anual, recolectada mensualmente de la estación más cercana al volcán Irazú, denominada "Estación 137 volcán Irazú", localizada a una altura de $3400 \mathrm{~m}$ s.n.m, y a menos de $2 \mathrm{~km}$ del cráter principal. Sin embargo, se debe tener en cuenta que en el 2007 no se contó con los datos de precipitación por parte del IMN. 


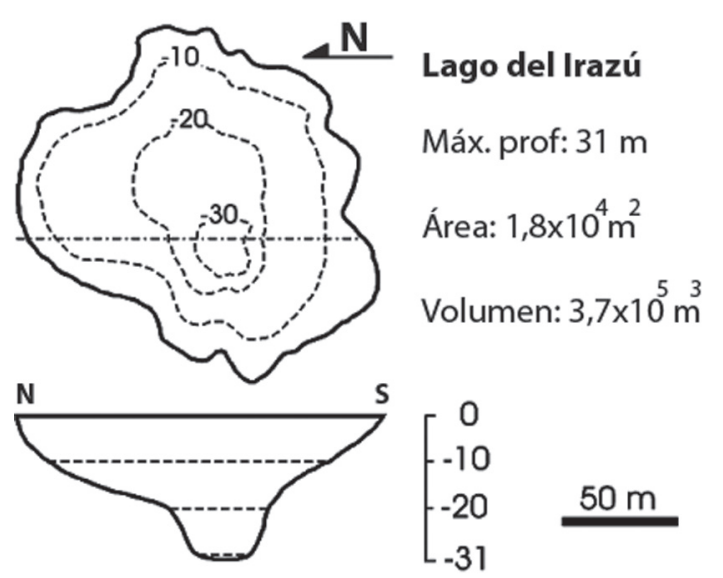

Fig. 2. Batimetría del lago del Irazú (modificado de Tassi et al., 2009).

Otro factor importante a considerar es la actividad sísmica que se presenta en las cercanías del Irazú, en un radio de aproximadamente $9 \mathrm{~km}$, ya que eventualmente podría afectar a la hidrología, la morfología, la velocidad en que ocurren los cambios y con ello, la laguna y sus modificaciones. Los datos se obtuvieron de la Red Sismológica Nacional (RSN), la cual posee una estación en las cercanías del volcán y monitorea cualquier tipo de actividad sísmica. No obstante, dentro de la información suministrada, no se indica la profundidad de cada uno de los eventos y no en todos los años está especificada la magnitud, por lo que no se puede afirmar que la profundidad o la magnitud de los sismos pueda tener en algunos casos una relación directa con los cambios producidos.

\section{SÍNTESIS HISTÓRICA DE LA ACTIVIDAD CRATÉRICA (1965-2012)}

El cráter del Irazú posee un diámetro de unos $1000 \mathrm{~m}$ y una profundidad de aproximadamente $200 \mathrm{~m}$. Está conformado por una alternancia de coladas de lava y rocas piroclásticas con paredes escarpadas hasta verticales. Basados en la recopilación bibliográfica desde el año 1965 hasta el 2012, se realizó una sinopsis en donde se relacionan los cambios del nivel y color de la laguna del Irazú. Este lapso de tiempo es tomado en consideración, dado que es precisamente en 1965 cuando el Irazú realiza su última actividad eruptiva importante. A partir de este suceso, la vigilancia de este macizo se vuelve más consistente y periódica, sumado a que se obtuvieron datos de diversas estaciones de medición meteorológica y sismológica para apoyar el análisis realizado con los otros parámetros considerados. Tal y como se aclaró previamente, también se consultó mucha información inédita o dispersa, entre ellas fotografías de diversas personas, notas de campo, y claramente la información sobre el estado de los volcanes de la RSN y el OVSICORI.

\section{Cronología de los hechos más relevantes}

En esta sección se realiza una investigación y síntesis de datos históricos relacionados con la coloración de la laguna, el nivel, los deslizamientos de las laderas, la sismicidad, actividad fumarólica que se ha presentado a lo largo del tiempo y que ha sido documentada, la cual se resume a continuación:

1965: En el mes de julio el fondo del cráter fue ocupado por una laguna (Kruschensky, 1972).

1973: En noviembre, el lago cubrió el fondo del cráter con un color verde y algunas fumarolas subacuáticas en el sector NW (Barquero, 1998; presente trabajo).

1976: El 18 de agosto se observó actividad fumarólica en los bordes de la laguna cratérica (Barquero, 1998).

1977: La laguna de aguas verdosas, que ocupaba el cráter principal, fue desapareciendo a inicios de 1977 (Paniagua \& Soto, 1986), más específicamente, desaparece en febrero, posiblemente por filtración por una fractura. El 3 de marzo se reactivó la actividad del Irazú con pequeñas erupciones de gases y ceniza (Alvarado, 1989). La laguna se formaba durante la época lluviosa y se secaba en la época seca (Barquero, 1998).

1978: Se observaron fumarolas subacuáticas (Alvarado, 1989).

1979: En febrero se observan deslizamientos dentro del cráter principal y materiales producto de la erosión. El cráter principal continúa rellenándose por materiales que se deslizan de las 


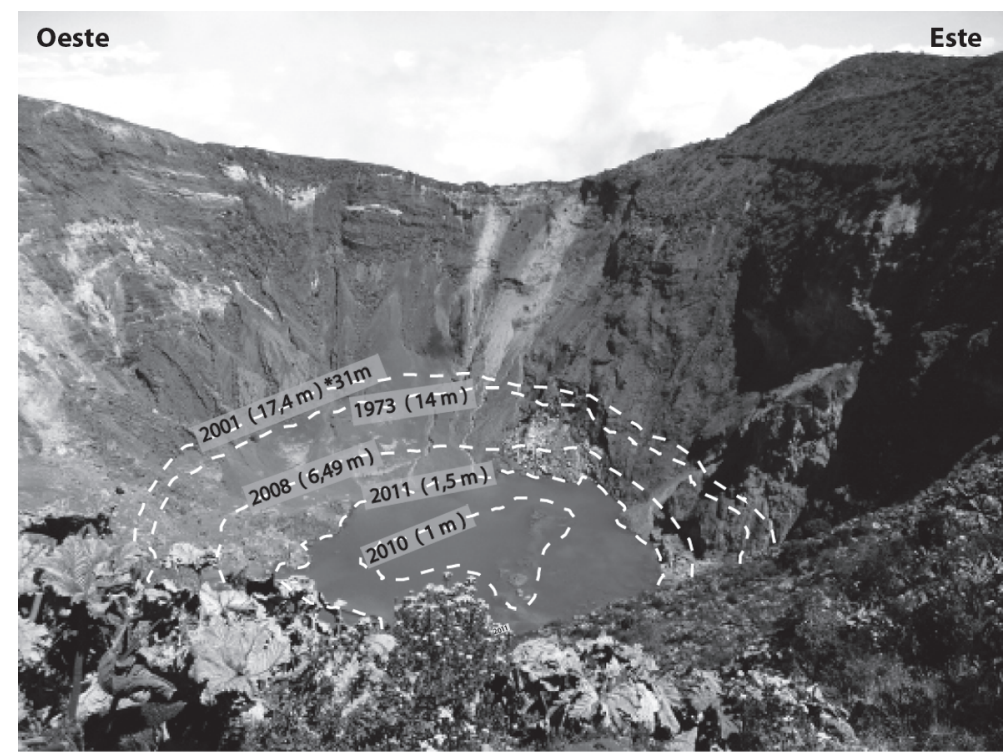

Fig. 3: Niveles promedio para referencia del nivel del lago, fotografía tomada el 03 de octubre del 2011. En el 2001 se registra una profundidad máxima de $31 \mathrm{~m}$ correspondiente al estudio batimétrico de Tassi et al. (2009).

paredes, los que son arrastrados por la intensa erosión. No se ha formado aún la laguna a pesar de las lluvias intensas de septiembre (OVSICORI, 1979).

1980: En la parte sur del cráter principal, se formó una grieta de E-W y una fractura ensanchada por aguas de escorrentía, mientras que en la pared W se presentan deslizamientos. En un descenso realizado en enero de este año, se comprueba que existen descargas de agua subterránea dentro del cráter y precipitación en los últimos meses del año (OVSICORI, 1980).

1981: En marzo todavía no se había formado la laguna mientras que el cráter principal empieza a producir material producto de la erosión (OVSICORI, 1981).

1982: En enero de 1982 el cráter estaba seco (Barquero, 1998), pero para marzo existía actividad de fumarolas subacuáticas con una temperatura entre $70^{\circ} \mathrm{C}$ y $90^{\circ} \mathrm{C}$ (Venzke et al., 2002), acompañado de sismicidad y de una pequeña colada de azufre dentro del cráter (McClelland et al., 1989).

1983: En junio se observan deslizamientos en la pared que divide el cráter principal con el cráter Diego de la Haya, asociado al enjambre de sismos del 4 de junio debido a la actividad de una falla local (OVSICORI, 1983).
1984: En septiembre se vuelve a formar una laguna blanquecina, sin actividad fumarólica, que posteriormente se tornó amarillenta-verdosa (mostaza), mostrando un tenue burbujeo en el sector NE por el escape de gases (Paniagua \& Soto, 1986; Alvarado, 1989; McClelland et al., 1989; Barquero, 1998).

1985: Actividad fumarólica en el flanco norte con temperaturas de $60^{\circ} \mathrm{C}$. La laguna que se empezó a formar en septiembre de 1984, permanecía hasta julio de 1985, con presencia de burbujas de la emisión de gases (Venzke et al., 2002).

1986: En septiembre, la laguna cratérica tenía pequeñas burbujas por el gas que emiten las fumarolas en el mes de septiembre (OVSICORI, 1986).

1987: A finales de junio se seca nuevamente la laguna (Alvarado, 1989).

1988: Debido a las intensas lluvias durante agosto y septiembre, se forman pequeñas lagunas verdes en el cráter principal y en el cráter Diego de la Haya. No se observaron signos de desgasificación fumarólica en las lagunas cratéricas (Venzke et al., 2002).

1989: Hay una pequeña laguna verde esmeralda que cambio a amarillento mostaza (Venzke et al., 2002; presente trabajo). 
1990: En abril desaparece la laguna cratérica (Alvarado, 2000).

1991: En junio se formó nuevamente la laguna en el ciclo de actividad de este año (Alvarado, 2000). Durante la segunda semana de junio, un grupo de fumarolas se formaron en el NE, N, NW, $\mathrm{y}$ en el $\mathrm{S}$ del cráter, con temperaturas de hasta $94^{\circ} \mathrm{C}$, similares a las fumarolas en el flanco $\mathrm{NW}$, que poseen una temperatura entre $80-90^{\circ} \mathrm{C}$. La laguna cratérica se empezó a llenar en la última semana de junio. Claramente, las aguas de la laguna, cerca de las fumarolas, poseían una temperatura y $\mathrm{pH}$ elevados $\left(91,0^{\circ} \mathrm{C}\right.$ y $\left.\mathrm{pH} 5,60\right)$. Pequeños deslizamientos ocurrieron en las paredes E, NE y SW (Venzke et al., 2002). En julio, la laguna verde turquesa, que continuaba aumentando en profundidad, cubriendo $2 / 3$ partes del fondo del cráter, con algunas fumarolas que se forman durante mitad de junio. El 17 de julio, la laguna posee $150 \mathrm{x}$ $100 \mathrm{~m}$, con una profundidad media de $2 \mathrm{~m}$. En los meses de agosto y septiembre, la laguna cratérica crece hasta cubrir casi todo el piso, con más de $150 \mathrm{~m}$ en diámetro. Un nuevo grupo de fumarolas aparecen en el cráter (Venzke et al., 2002). A mediados de noviembre, la laguna verdosa humeaba en partes, y presentó precipitados de hidróxidos de hierro en el fondo de la misma (observado en partes menos profundas de color rojizo). Además, se observa la llegada de lodo y arena posiblemente debido a un periodo de lluvias o por la actividad de algas termofílicas que colonizaron la parte somera de la laguna. Entre setiembre 1991 y abril de 1992, las temperaturas variaron entre 25,90 y $34,40^{\circ} \mathrm{C}$ y el $\mathrm{pH}$ entre 2,85 y 3,86 (Barquero et al., 1992; presente trabajo). En diciembre de 1991 la laguna posee una temperatura de $26,7^{\circ} \mathrm{C}$ y un $\mathrm{pH}$ de 3,0. El nivel sube $40 \mathrm{~cm}$ desde octubre y el radio de la laguna cratérica crece en $5 \mathrm{~m}$ adicionales (Venzke et al., 2002).

1992: En enero, se midió y el nivel de la laguna había aumentado un metro, asociado a las lluvias fuertes de finales de noviembre del año anterior. El nivel de la laguna disminuye unos centímetros en comparación con el mes de febrero, con temperaturas de $27,0-30,70{ }^{\circ} \mathrm{C}$ y pH de $3,23-3,86$. En junio, se informa la aparición de una laguna de color verde limón a verde blancuzca con temperaturas de $28^{\circ} \mathrm{C}$ y $\mathrm{pH}$ de 4,9 . En julio, el nivel de la laguna, color verde turquesa, aumenta su nivel, pero en septiembre, presenta un color verde oscuro, y su nivel disminuye o aumenta con las variaciones de la lluvia. En diciembre, el nivel de la laguna continúa subiendo, cubriendo casi todo el fondo del cráter (Barquero, 1998; Venzke et al., 2002; presente trabajo).

1993: En febrero, la laguna cratérica presenta un color de verde turquesa a amarillo claro, aumentando $12 \mathrm{~cm}$ su nivel; solo en febrero disminuyó $40 \mathrm{~cm}$ su nivel. En marzo, se muestra actividad fumarólica al $\mathrm{N}$ y NW del cráter y la laguna permanece de color verde. El nivel de la laguna fluctúa unos 10-15 cm dependiendo de la precipitación. Las paredes E y SE presentan derrumbes. Desde septiembre hasta octubre, el nivel de la laguna aumenta $40 \mathrm{~cm}$ y en octubre presenta un color verde esmeralda. En noviembre, el nivel de la laguna aumenta $20 \mathrm{~cm}$ y posee fumarolas subacuáticas al N y SE del cráter (Venzke et al., 2002). Su temperatura varió entre 17,4 y $35^{\circ} \mathrm{C}$, con $\mathrm{pH}$ entre 5,50 y 5,80 (Barquero, 1998; presente trabajo).

1994: En noviembre el nivel de la laguna disminuyó $40 \mathrm{~cm}$ durante la época seca. Posee color verde turquesa a verde oscuro con fumarolas subacuáticas en la parte norte y SE. Durante la época lluviosa, el nivel del lago cubría casi todo el fondo del cráter. Se observaron deslizamientos en la pared N, E y SW, mostrando en la pared norte puntos con actividad fumarólica. Entre enero y marzo se registron 884 sismos locales. Posee una laguna tibia $\left(25^{\circ} \mathrm{C}\right)$ y acidez moderada $(\mathrm{pH}$ 5,5 ). Las dos líneas de nivelación trigonométricas ubicadas al S y al SE del cráter principal, mostraron una deflación promedio de $13 \mu \mathrm{rad} /$ año. Un sector de la red geodésica de la cima registró una contracción de 7 ppm/año, y dilatación areal de 10 ppm/año. Para el día 8 de diciembre a las 04:48 GMT, se produce un deslizamiento lateral en el flanco NW del volcán (Barquero et al., 1995; Mora, 1997; Venzke et al., 2002).

1995: El lago cubre el fondo del cráter con un color entre amarillo a amarillo verdoso. En la orilla N, NW, W, SW se presentó un burbujeo en forma permanente y hacia la pared N, E y SE se producen deslizamientos pequeños. En marzo, la laguna cratérica posee una temperatura entre 18 
y $23^{\circ} \mathrm{C}$, mientras que su nivel aumenta $1 \mathrm{~m}$ con respecto a los últimos niveles. La laguna posee un estimado de 430 millones de $\mathrm{m} 3$ de agua. En abril disminuye $50 \mathrm{~cm}$. En junio, el color de la laguna es amarillo oscuro y cubre todo el piso del cráter, mientras que las paredes del cráter continúan deslizándose hacia la laguna en las laderas N, E y SE (Mora, 1997; Venzke et al., 2002).

1996: Durante el mes de enero el nivel del lago se mantuvo cubriendo todo el fondo del cráter con un color amarillo oscuro. En la orilla N, NW y SE se presentaba un burbujeo en forma permanente, mientras que las paredes $\mathrm{N}, \mathrm{SW}$ y $\mathrm{W}$, presentaron deslizamientos pequeños (Mora, 1997; Venzke et al., 2002).

1997-2000: De febrero a marzo de 1998, el color del lago era verde claro. Las fluctuaciones del nivel de la laguna se notaron desde diciembre hasta marzo en menos de un metro. Durante mayo del 1998, la laguna presentó un color amarillo pálido y presentaba pequeños deslizamientos al N, E y W. En el año 1999, de febrero a marzo, su color era amarillo claro, pero en mayo se torna verde, con un constante burbujeo. Durante mayo del 2000, el nivel del lago disminuyó $50 \mathrm{~cm}$ y el color de la laguna era amarillo/verde. Una cantidad significativa de algas cubría la superficie. En las paredes E, N y W del cráter continúan deslizándose hacia el cráter. La temperatura de la laguna cratérica es $18^{\circ} \mathrm{C}$. En junio, la superficie de la laguna cratérica aumenta $40 \mathrm{~cm}$ en comparación al mes de mayo, el color es verde y su superficie está cubierta de algas. La pared NE del cráter continuaba deslizándose, cubriendo algunas fumarolas (Venzke et al., 2002).

2001: Se mantiene estable el nivel del lago con un color verde (Venzke et al., 2002). Tassi et al. (2009) indica que la profundidad promedio de la laguna fue de $14,7 \mathrm{~m}$ para el mes de octubre, sin embargo, indican una profundidad máxima de 31 $\mathrm{m}$ para ese mismo año.

2002: El nivel del lago cubría el fondo del cráter con un color amarillo verdoso. En la orilla NW y SE, se mantuvo un burbujeo. Para febrero, la laguna presentaba un color amarillo y descendió $40 \mathrm{~cm}$. En marzo descendió el nivel del lago 5,6 $\mathrm{m}$ y presentaba un color turquesa con partículas de azufre. Para abril, el color se tornó amarillo y el nivel del lago descendió 1,40 m. En septiembre se observan deslizamientos en la pared $\mathrm{W}$ y un descenso del nivel de la laguna debido a marcas observadas a simple vista y con binoculares de antiguos niveles del agua. Se observaron costras $\mathrm{y}$ algas que flotaban en la orilla del lago. Se registran 3 a 6 sismos mensuales, con un promedio de 4 sismos por mes (Mora, 2003).

2003: El nivel del lago se mantuvo cubriendo todo el fondo del cráter con un color amarillo verdoso a mostaza. En la orilla NE, N y NW existía un burbujeo. Para febrero, presentaba un color amarillo fuerte, posteriormente cambia a rojizo, debido al aporte de material alterado proveniente de deslizamientos en las paredes $\mathrm{E}$ y NE (OVSICORI, 2003). Según Mora (2005), el lago presentó un cambio de coloración el 9 de febrero del 2003, de verde claro a un color amarillo-mostaza. Se apreciaron manchas color café-rojo que flotaban y que tenían una forma oblonga alargada de varios metros de largo, que desaparecían y se formaban en minutos. En julio del 2003, se apreció un descenso en el nivel de la laguna, dejando estructuras de laminación paralela que se ubican en el lado N. En octubre del 2003, se dio un aumento en el nivel, además de un leve burbujeo en la laguna hacia su lado $\mathrm{N}$, cerca de la pequeña península conocida por tener pequeñas liberaciones de gases y mantener una temperatura superior a $\operatorname{los} 30^{\circ} \mathrm{C}$ (Mora, 2005; Van der Laat, 2009).

2004: El nivel del lago se encontraba cubriendo todo el fondo del cráter con un color verde blancuzco con costras de hidróxidos de hierro en el litoral (febrero) a amarillo verdoso, que cambió a verde turquesa de abril a octubre. En la orilla NW y SE, se mantuvo el burbujeo. En abril el nivel del lago descendió $50 \mathrm{~cm}$ con respecto al mes de enero (Mora, 2005; Van der Laat, 2009).

2005-2006: El nivel del lago cubría todo el fondo con un burbujeo en las orillas NE, NW, N y SE y con un intenso color verde turquesa (Van der Laat, 2009).

2007: El nivel del lago cubría todo el fondo con un burbujeo en las orillas NE, NW, N y SE. Desde marzo hasta septiembre, se registró un descenso del nivel de $3,87 \mathrm{~m}$, a pesar de la época lluviosa; además, la coloración del agua cambió de amarillo a verde. Para diciembre, el nivel del 
lago posee el nivel más bajo de los últimos dos años con un color amarillo verdoso (Van der Laat, 2009; presente trabajo).

2008: El nivel del lago se mantuvo cubriendo solamente su fondo con un burbujeo en las orillas NE, NW, N y SE, con un color verde turquesa en marzo y abril, que cambio a verde amarillento pálido en septiembre, con un nivel bajo de la laguna. En marzo se reporta un descenso del nivel del lago de 4,58 $\mathrm{m}$ con respecto al nivel medido el 20 de septiembre del año anterior. Para el mes de mayo se recupera parte del nivel del lago debido al inicio del periodo lluvioso, pero mantuvo su color amarillo verdoso. A partir de abril, se observan un par de islotes, producto del bajo nivel del agua y los materiales de deslizamientos que han ocurrido en años recientes (Van der Laat, 2009; presente trabajo).

2009: El nivel de la laguna cratérica en enero aumentó con respecto a diciembre del 2008; éste presentó un color verde turquesa con una orilla de color amarillo. En marzo descendió el nivel del lago con respecto al nivel de febrero mientras que en septiembre se da un descenso de 3,96 m con respecto a marzo del 2008. Presentaba bordes de sedimentos rojizos (Van der Laat, 2009; presente trabajo).

El 7 de octubre de 2009 el nivel del lago ha descendido $3,38 \mathrm{~m}$ y la temperatura fue de $16^{\circ} \mathrm{C}$. En la orilla NW quedan $70 \mathrm{~cm}$ por evaporarse y/o infiltrarse. Las áreas cubiertas por lodo y arcilla cubren prácticamente todo el fondo, mismas que históricamente se habían mantenido bajo el agua. En algunos sectores se observan parches de vegetación acuática en proceso de descomposición. A finales de 2009, se informó sobre un inusual descenso del nivel, sin razón aparente y sin proceso de reversión. Desde cualquier ángulo el nivel de la laguna se veía reducido y de un color verde claro, casi transparente (Fernández \& Duarte., 2010a, b).

2010: El lago presentaba un color turquesa pero su nivel continúa descendiendo en enero y febrero hasta estar a punto de secarse; en la parte central se observan isletas de roca y sedimentos de color rojizo. El nivel descendió entre el 7 de octubre del 2009 y el 12 de marzo del 2010, restando únicamente $1,40 \mathrm{~m}$ para secarse completamente, hecho que se registró en mayo, cuando se secó completamente la laguna. En agosto, en el fondo del cráter se empieza a formar nuevamente el lago $\mathrm{y}$ en octubre se vuelve a secar. En noviembre se vuelve a formar el lago con un tono verde claro y sigue recuperándose hasta diciembre cuando se torna de color turquesa (presente trabajo).

2011-2012: En noviembre del 2011, la laguna presenta un color turquesa con algunos tonos amarillos en las orillas, donde las isletas de material y sedimentos se continúan observando por encima del nivel de la laguna, aunque con un nivel muy bajo, en vías de desecación a raíz de un periodo de pocas lluvias (presente trabajo).

\section{DISCUCIÓN}

A continuación se realiza un análisis integral de los parámetros que afectan el nivel de la laguna cratérica, así como sus cambios de coloración, con el fin de entender cada uno y la relación entre ellos, de modo tal, que nos permita producir un análisis de las condiciones que afectan la laguna y la modifican. Los parámetros se investigaron en un rango que comprende los años desde 1965 hasta el 2012, dado que se quiso estudiar los cambios en la laguna formada después de la última gran actividad eruptiva del volcán Irazú. Sin embargo, tal y como se ha dicho en varios apartados, se poseen muchas limitantes en la base de datos.

En la figura 4 se han graficado los parámetros que se posiblemente podrían afectar la laguna del volcán Irazú directamente, con el fin de visualizar si existe alguna relación entre ellos, que puedan probablemente estar influenciando los cambios de color y profundidad en la laguna. Se puede observar que los datos no son continuos, ya que los registros no tenían un rango amplio de tiempo, o bien del todo no se encontraban en las bases de datos debido a que el equipo se encontraba dañado y no se pudieron tomar los registros, como es el caso del año 2007 con los datos de precipitación.

Resulta importante señalar, que en la sección de datos, lo que corresponde con la profundidad del lago (Fig. 4a), se maneja con un margen de error moderado, dado que la información a lo largo del tiempo fue reportada de forma relativa y no 

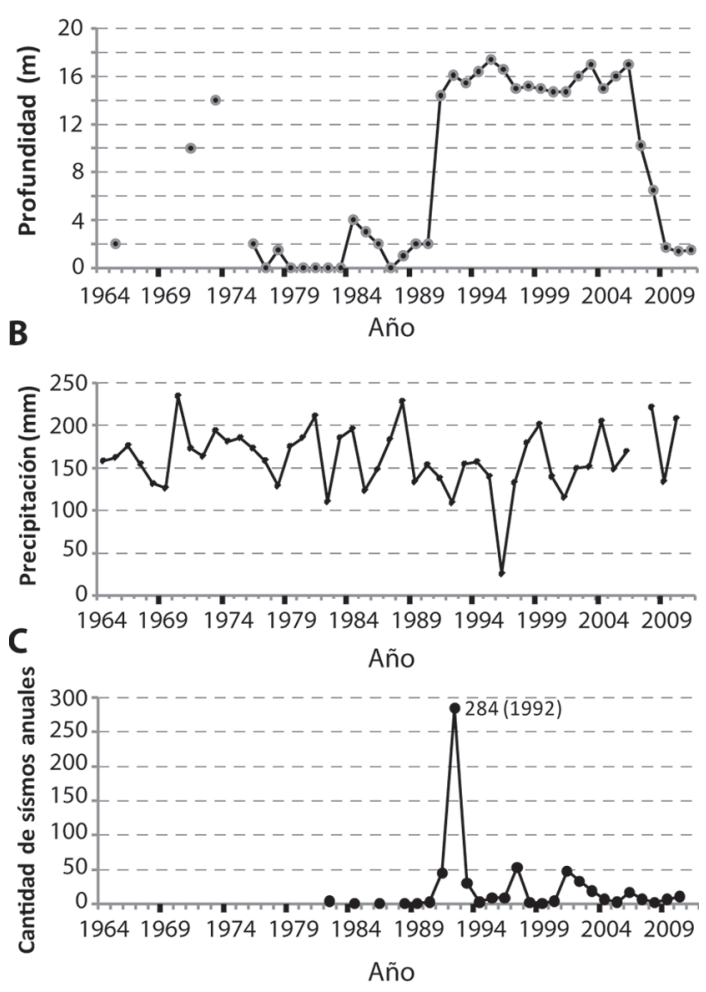

Fig. 4: Comparación de la tendencia en (A) la profundidad del lago (diversas fuentes citadas en el texto). (B) Precipitación IMN y (C) sismicidad RSN.

se contó con datos absolutos de la profundidad del lago, con algunas excepciones como el caso del estudio de Tassi et al. (2009). Para el desarrollo de este apartado, se contó con un total de 48 valores, de los cuales 30 son tanto medidas relativas, de las cuales no hay registros de las metodologías empleadas para determinar el valor registrado; y absolutas, como las mediciones batimétricas de Tassi et al. (2009); 9 datos son valores relativos por medio de comparación entre fotografías históricas de la laguna y 9 no tienen un valor de profundidad medida.

\section{Precipitación versus profundidad}

La precipitación en el volcán Irazú muestra sus variantes en dependencia de la época en la que nos encontremos, ya sea, en la época lluviosa (mayo-noviembre) o en la época seca (diciembreabril), así como factores externos que pueden afectar el clima tales como temporales, temporada de huracanes, el fenómeno del Niño o la Niña. Según los datos proporcionados por el Instituto Meteorológico Nacional (Fig. 4b), el valor promedio de precipitación de la estación meteorológica del volcán Irazú es de 160 mm anuales, pero en épocas donde la precipitación es importante, se registran valores de hasta $228 \mathrm{~mm}$ anuales y en temporadas de escasas lluvias se presentan valores de $26 \mathrm{~mm}$ anuales.

Al tomar en cuenta el parámetro de la precipitación, se debe considerar también la dinámica hidrogeológica del sistema en los alrededores de la laguna, porque solo así se puede determinar la interacción entre las lluvias, la infiltración y el transito del agua en el sistema, comprendiendo así, cuál sería el tiempo de respuesta que daría un fuerte aguacero o un prolongado temporal reflejado en un aporte a la laguna. Basados en los datos recopilados solo para los años 2008 y 2010, se tienen valores de nivel en diferentes meses del año, coincidiendo algunos con la época seca y otros en la época lluviosa. Con estos datos se puede tener una aproximación de la influencia que tiene la precipitación en la época seca y la época lluviosa para estos años sobre la laguna, y de esta forma determinar si hay alguna conexión entre el parámetro precipitación y el parámetro profundidad (Fig. 5).

Para el año 2008, se observa como durante la época seca (diciembre-abril) la precipitación se mantiene en un promedio inferior a los $100 \mathrm{~mm}$, la laguna presenta una profundidad de 5,64 m. Cambiando las condiciones a época lluviosa en los meses de mayo a noviembre, se puede observar cómo cambia el nivel de la laguna incrementándose en menos de un metro su profundidad. De la Fig. 5 se puede especular que este cambio de nivel se da justo en el cambio de época seca a lluviosa, por lo que se podría pensar también en que no hay un tránsito de duración importante entre el agua de lluvia y la incorporación a la laguna después de pasar por el sistema hidrogeológico, y que la recarga de la laguna se da en menos de un mes al cambiar las condiciones en la precipitación. Pero al tomar en cuenta la Fig. 6, se nota como para el año 2010 sucede un fenómeno completamente inverso al observado en el 2008, teniendo 


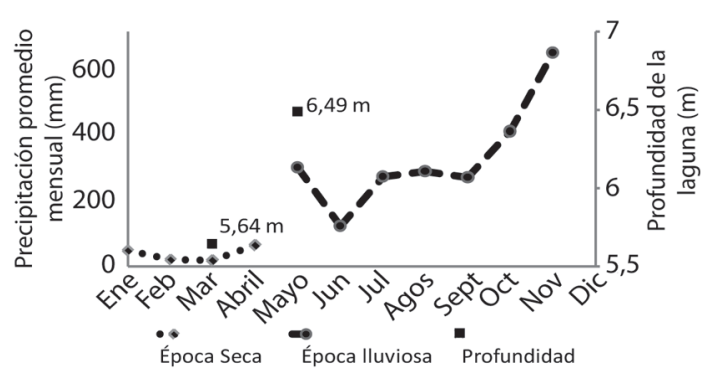

Fig. 5: Variación de la profundidad con respecto a la precipitación promedio mensual en el 2008.

un cambio de nivel de 5,64 m, justamente en el cambio de época seca a época lluviosa; incluso se puede ver que en la época lluviosa la laguna fluctúa entre estar completamente vacía y alcanzar a lo máximo $1 \mathrm{~m}$ de profundidad.

De una manera más amplia, la Fig. 4, muestra una tendencia a disminuir el nivel del lago entre los años 1973, 1979 y 1982, mientras que la precipitación se mantuvo en un promedio anual de $170 \mathrm{~mm}$ en este periodo, dándose una variación apreciable en el nivel del lago. Otro ejemplo, donde se nota que la precipitación no siempre influye en el nivel del lago, se observa en 1987, donde se seca la laguna, aunque la precipitación es mayor a $180 \mathrm{~mm}$ anuales, manteniéndose dentro del promedio de precipitación anual normal.

También, en el periodo de 1990-1996, se da una variación del nivel pero sin que se seque la laguna, dado que se mantiene entre un nivel alto de $16-18 \mathrm{~m}$ de profundidad, mientras que la precipitación fluctúa entre $153 \mathrm{~mm}$ y $25 \mathrm{~mm}$ anuales, lo cual no parece afectar el nivel del lago aunque la precipitación es relativamente baja. Un patrón similar se observa entre 1997-2006, ya que el nivel se mantiene constante y alto, aunque la precipitación varía en un promedio bajo de $160 \mathrm{~mm}$ anuales, con un pico máximo en el año de 1999 de $200 \mathrm{~mm}$.

Observando el patrón anual de la precipitación desde un punto de vista detallado o bien amplio, se puede constatar que al parecer no hay una relación directa con los cambios de nivel de la laguna. No obstante, debe tomarse en consideración que las pequeñas fluctuaciones si pueden ser producto de los cambios de época, como en los meses donde se presenta mayor precipitación, claramente puede aumentar el volumen de la laguna y niveles bajos corresponden en muchas ocasiones con la época seca.

\section{Movimientos de ladera}

Prácticamente, en todos las laderas internas del cráter Principal (N, NW, W, SW y E) se han documentado a lo largo del tiempo deslizamientos de diverso tamaño. Se observó al descender el lago, intercalaciones de diferentes granulometrías entre ellas arenas, gravas y arcillas con estratificación paralela planar. Estas afloran tanto en la parte del fondo del cráter como en las zonas periféricas al fondo del cráter, siendo evidencia de sedimentación subacuática de material que cayó desde las laderas, estas secuencias presentan en algunas zonas registros de grietas de desecación que refuerzan la teoría de una sedimentación subacuática.

Sin duda, los deslizamientos dentro del cráter hacen variar el nivel del lago, aportando volumen importante de material detrítico a la zona donde está emplazada la laguna, desplazando la parte líquida y rellenando el fondo. Aún así, no se ha cuantificado su efecto directo sobre el nivel de la laguna por causa de desprendimiento de grandes bloques.

\section{Color del lago}

En el Cuadro 1, se muestran los datos que hacen referencia al color de la laguna, sustraídos del apartado de síntesis histórica de la actividad del cráter del Irazú tratado anteriormente. En esta tabla se logran apreciar varios cambios de color de la laguna en un mismo año, en comparación con otros periodos.

Aparentemente, el color amarillo verdoso del lago está asociado a niveles altos de la laguna con poca actividad fumarólica subacuática, pocos deslizamientos y posiblemente un mayor desarrollo de algas. El color puede cambiar desde tonos verdes hasta colores amarillentos, cuando existe una actividad fumarólica más intensa que enriquece en sales las aguas. Se observa que cuando 


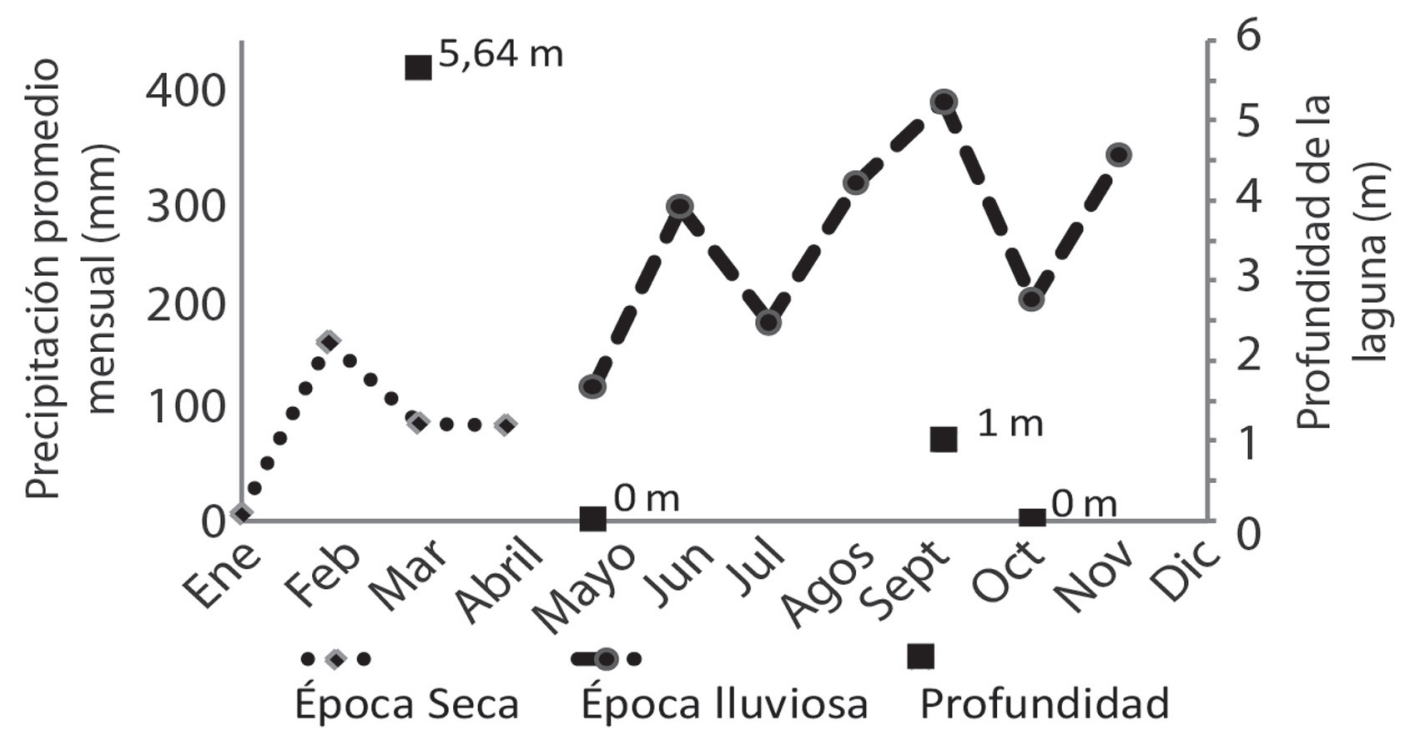

Fig. 6: Variación de la profundidad con respecto a la precipitación promedio mensual del año 2010.

el agua de la laguna es relativamente tibia y con un $\mathrm{pH}$ ácido $\left(26,7-37^{\circ} \mathrm{C}\right.$ y $\left.\mathrm{pH} 3,0-4,9\right)$, tal como ocurrió en los años 1991-1992, la actividad fumarólica subacuática era muy intensa y los colores de la laguna variaban entre verde turquesa a verde oscuro, con precipitados rojizos de hidróxidos de hierro en sus bordes. Los deslizamientos, como aquellos presuntamente ocurridos a inicios del 2003, promovieron el cambio de color de amarillo verdosa hasta mostaza a rojizo por la incorporación de sedimentos finos y ferruginosos (hidróxidos de hierro). Los colores turquesa posiblemente están asociados a sílice coloidal y a las sales ferrosas. Sin embargo, la información histórica y los pocos análisis químicos de la laguna y de las algas que crecen en su interior, no permiten mejores conclusiones y por ello, se requiere estudios más específicos en el futuro.

\section{Sismicidad}

En 1992 se presentaron una gran cantidad de sismos y la laguna comenzó un descenso del nivel del lago, sin embargo, al analizar el periodo entre 1991-1997, se observa un punto máximo contrastado por uno mínimo de sismicidad y aún así, la laguna no presenta una variación importante manteniéndose prácticamente constante hasta el 2007 (Fig. 4c). No obstante, la actividad sísmica podría tener una contribución sobre la filtración de agua hacia niveles inferiores, produciendo reducción del nivel de la laguna o el llenado de la misma, ya que los sismos pueden influir en las fracturas que se encuentran en el fondo del lago, ya sea ensanchándolas, prolongándolas o bien bloqueándolas. Por ejemplo, en el enjambre del Irazú de principios de 1991, afectó el sistema hidrotermal mediante la aparición de fumarolas intracratéricas y la formación de la laguna en julio y agosto, después de estar seco el cráter desde enero hasta mayo (Barquero et al., 1992).

\section{INTERPRETACIÓN}

En esta sección se analizan los parámetros antes mencionados de manera conjunta de modo tal que se puedan generar varias hipótesis y modelos sobre las variaciones en la laguna cratérica, que ayuden a comprender los diferentes fenómenos observados. Como se trató en la sección anterior, los fenómenos que suceden en la laguna no dependen de un solo factor, sino de la interacción de todos en conjunto. 
Cuadro 1

Variación de la coloración de la laguna intra-cratérica del cráter principal del Irazú

\begin{tabular}{|c|c|c|c|c|c|}
\hline Años & Color & Años & Color & Años & Color \\
\hline $\begin{array}{c}1973 \text { y } \\
1977\end{array}$ & Verde & $\begin{array}{l}1994 \\
\text { 『 } \Delta\end{array}$ & Verde turquesa a verde oscuro & $\begin{array}{l}2002 \\
\text { ㄴ } \Delta\end{array}$ & Amarillo verdoso, turquesa \\
\hline 1984 & $\begin{array}{l}\text { Amarillo verdoso y } \\
\text { blancuzca a mostaza }\end{array}$ & $\begin{array}{l}1995 \\
\text { Ф } \Delta\end{array}$ & $\begin{array}{l}\text { Amarillo verdoso, amarillo } \\
\text { oscuro a verde oscuro }\end{array}$ & $\begin{array}{l}2003 \\
\text { ㄴ } \Delta\end{array}$ & $\begin{array}{l}\text { Amarillo verdoso, amarillo } \\
\text { fuerte (mostaza), café y rojizo }\end{array}$ \\
\hline 1988 & Verde & $\begin{array}{l}1997 \\
\text { } \Delta\end{array}$ & $\begin{array}{l}\text { Amarillo oscuro cambiando a } \\
\text { claro hasta verdosa }\end{array}$ & $\begin{array}{l}2004-2005 \\
\quad \text { } \Delta\end{array}$ & $\begin{array}{l}\text { Amarillo verdoso a verde } \\
\text { intenso }\end{array}$ \\
\hline 1989『 & $\begin{array}{l}\text { Verde esmeralda a mo- } \\
\text { staza }\end{array}$ & $\begin{array}{c}1998 \\
\text { ॠ }\end{array}$ & Verde claro, amarillo pálido & $\begin{array}{l}2007 \\
\text { ㄴ } \Delta\end{array}$ & Amarillo a verde \\
\hline 1991『 $\Delta$ & Verde turquesa & $\begin{array}{l}1999 \\
\text { Ф } \Delta\end{array}$ & $\begin{array}{l}\text { Amarillo claro a verde azu- } \\
\text { lado }\end{array}$ & $\begin{array}{l}2008 \\
\text { ए } \Delta\end{array}$ & $\begin{array}{l}\text { Verde turquesa a amarillo } \\
\text { verdoso }\end{array}$ \\
\hline 1992匹 $\Delta$ & $\begin{array}{l}\text { Verde turquesa y verde } \\
\text { oscuro }\end{array}$ & $\begin{array}{l}2000 \\
\text { Ф } \Delta\end{array}$ & Amarillo verdoso, verde & 2009 & Turquesa a amarillo (orilla) \\
\hline 1993『 $\Delta$ & $\begin{array}{l}\text { Turquesa (verde es- } \\
\text { meralda) a verde ama- } \\
\text { rillo claro }\end{array}$ & $\begin{array}{l}2001 \\
\text { ㄴ } \Delta\end{array}$ & Verde a amarillenta & 2010 & Turquesa verde claro \\
\hline \multicolumn{4}{|c|}{$\Delta$ Fumarolas subacuáticas 『 Deslizamientos } & 2011-2012 & $\begin{array}{l}\text { Turquesa con tonos amarillos } \\
\text { (orillas) }\end{array}$ \\
\hline
\end{tabular}

\section{Etapa 1: Sedimentológica}

La laguna en el cráter principal ha estado sujeta a cambios en su nivel a lo largo del tiempo. Aunque no se posee el dato exacto del surgimiento de la laguna, para el año 1977, Van der Bilt informa una desaparición de la laguna, lo cual implica que en el lapso desde 1965 a 1977 tuvo que haberse formado la laguna en algún momento (Alvarado, 1989).

La sedimentación presente en el lago es producto de la escorrentía y deslizamientos; el primero aporta componentes de grano fino y el segundo aporta granulometrías desde limos arcillosos hasta bloques métricos. Podemos suponer que en los sectores proximales de los deslizamientos se depositan elementos de varios tamaños, dominando los bloques y granulometría media, pero conforme se pasa a la parte distal, la granulometría es de menor tamaño, dominando las arenas, limos y arcillas. Las intercalaciones de estas últimas granulometrías, pueden actuar como impermeabilizantes en el fondo de la laguna.
Las visitas al lago del volcán Irazú, confirmaron este tipo de depósitos en forma de delgadas capas de arcillas de color café claro intercaladas con cenizas (Fig. 7). A lo largo del fondo del cráter y hacia sus paredes, se encontraron varios afloramientos con las mismas características descritas anteriormente, incluso en algunos sectores erosionados dejaban al descubierto antiguas secciones lacustres con grietas de desecación, evidencia de niveles mayores del lago (Fig. 8).

En general, si se toma en cuenta que los deslizamientos ocurren en toda la periferia interna del cráter, se produce entonces una gradación de sedimentos donde los componentes finos se concentran en la parte central del lago y conforme se avanza hacia las paredes, la granulometría aumenta de tamaño. Aunado a esto, se tiene el hecho de que la laguna ha tenido variaciones en su profundidad, tiene como consecuencia que los sedimentos de distintas granulometrías se distribuyan tanto cerca del centro del cráter como en la periferia del fondo del cráter, así se tienen zonas impermeables y permeables a distintos niveles. 


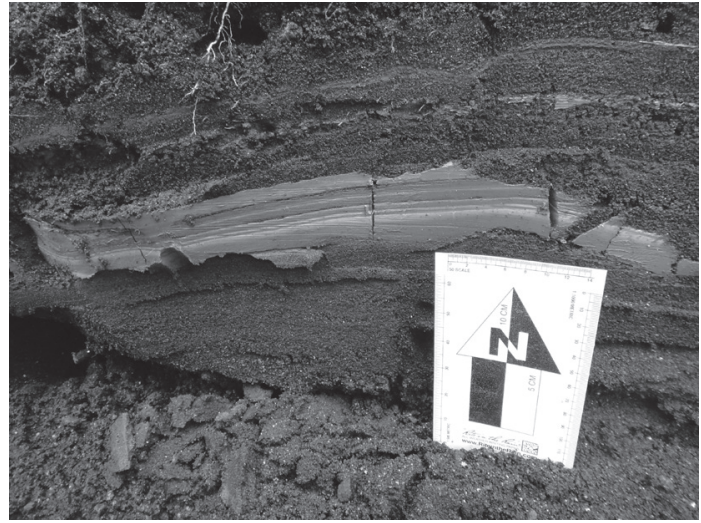

Fig. 7: Capas arcillosas y arenosas presentes en el fondo del lago. De abajo hacia arriba se observan cenizas, arcillas, grietas de desecación y arenas finas con intercalaciones milimétricas de arcilla.

Otro mecanismo, por el cual los sedimentos del fondo del lago pueden ser influenciados, es por la ocurrencia de la caída de bloques, que pueden aportar sedimentos de tamaños métricos al fondo del lago, provocando una deformación en los sedimentos, lo que resulta en grietas en el fondo de la laguna, afectando en ocasiones su apariencia, como por ejemplo su color, así como la ocurrencia del drenaje de la laguna ya que se rompe el sello impermeable.

\section{Etapa 2: Infiltración por contracción, hidro- fracturación o disolución}

Cuando la laguna presenta una disminución en el nivel, se puede argumentar que todo el volumen o la mayor parte de él, es conducido por una red de fracturas (estrechas y ensanchadas) que se localizan distribuidas por todo el fondo del cráter y quizás estas drenan hacia la ladera norte del volcán (Fig. 9). Estas fracturas, podrían haber sido rellenadas con arcillas y sales producto de la alteración hidrotermal durante los eventos de fumarolas en la laguna, actuando como reguladores del paso de su contenido. Para que se cumpla este fenómeno, se puede pensar en varios escenarios. El primero consiste en que las arcillas, dentro de

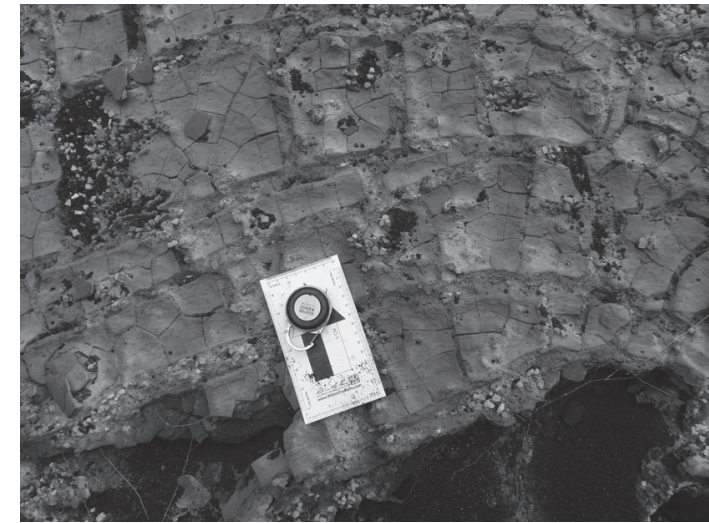

Fig. 8: Grietas de desecación, expuestas por erosión de niveles lacustrinos previos.

las fracturas, se contraen cuando hay poco aporte de agua desde la laguna y se dilatan cuando hay un aporte de agua de la laguna. Otro de los escenarios corresponde con la apertura de estas fracturas por movimientos sísmicos (hidrofacturación), resultando en bloqueos de algunos sectores y ensanchamiento en otros, dando paso a fluidos que podrían disolver las sales precipitadas en las fracturas, lo que provocaría un drenaje de la laguna y consecuentemente, el descenso en el nivel de la misma.

En una serie de visitas de campo realizadas en marzo del 2011 por el Grupo Espeleológico Anthros, se lograron ubicar y explorar dos cavidades en el sector NW del volcán Irazú. Estas cavernas presentan una orientación dominante N-NW. Dicha orientación coincide con la falla Irazú, la cual ha estado activa desde 1990 y se le atribuye la actividad freática en el flanco NW de 1994. Al relacionar el rumbo de las cuevas con los elementos estructurales cercanos, se puede hipotetizar una relación entre la orientación de las cavernas, las fallas que afectan el volcán y por consiguiente, una relación entre las fracturas, la laguna y la caverna. Estas cuevas son mencionadas en documentos como una estructura producto de una explosión lateral en diciembre de 1994.

Se propone, que cuando la laguna está en proceso de drenado, estas cavernas junto con un sistema de fracturas, canalizan el contenido de la laguna, depositándola finalmente en la naciente 
del río Sucio y lugares aledaños, ubicados al pie de la ladera escarpada donde se encuentra la cueva.

Al pie de las paredes internas de la caverna, se observaron canales de escorrentía superficial de al menos $3 \mathrm{~m}$ de ancho por $15 \mathrm{~m}$ de largo y $30 \mathrm{~cm}$ de profundidad emplazados en materiales limo-arenosos. El día de la visita, estos canales se encontraban secos, pero presentaban intactas las evidencias de un flujo. En la caverna, se observaron además, formaciones cristalinas de diferentes sales precipitadas tanto en las paredes como en el piso y formaciones de estalactitas de hasta $50 \mathrm{~cm}$ de largo de un color azul turquesa translucido muy característico, localizadas en ciertos sectores de la cueva, aprovechando las fracturas en el techo. Las estalactitas se encontraban aún en crecimiento (goteando) el día de la observación. Se podría pensar que todos estos minerales precipitados provienen de las fumarolas aledañas, pero también podrían estar conectadas con los minerales y fluidos provenientes de la laguna, o ambos (Fig. 9).

\section{CONCLUSIONES}

La aparición de la laguna en 1965, justamente cuando finaliza la última actividad eruptiva importante, es demasiado rápido para lograr formar una capa sello que realmente impermeabilice la laguna. La capa sello probablemente no está del todo formada y es muy frágil desde el punto de vista hidráulico. Por lo que esto es un facilitador del comportamiento intermitente de la laguna.

La condición de la laguna cratérica del Irazú, es afectada por distintos factores como movimientos de ladera, sismicidad, actividad hidrotermal y precipitación, esta última aunque es poco influyente es parte del sistema hidrotermal presente en el cráter.

La precipitación por sí sola, no demostró ser un factor determinante para el cambio del nivel del la laguna del volcán Irazú, sin embargo, si posee una influencia significativa, especialmente en eventos de precipitación importante o periodos prolongados de sequía.
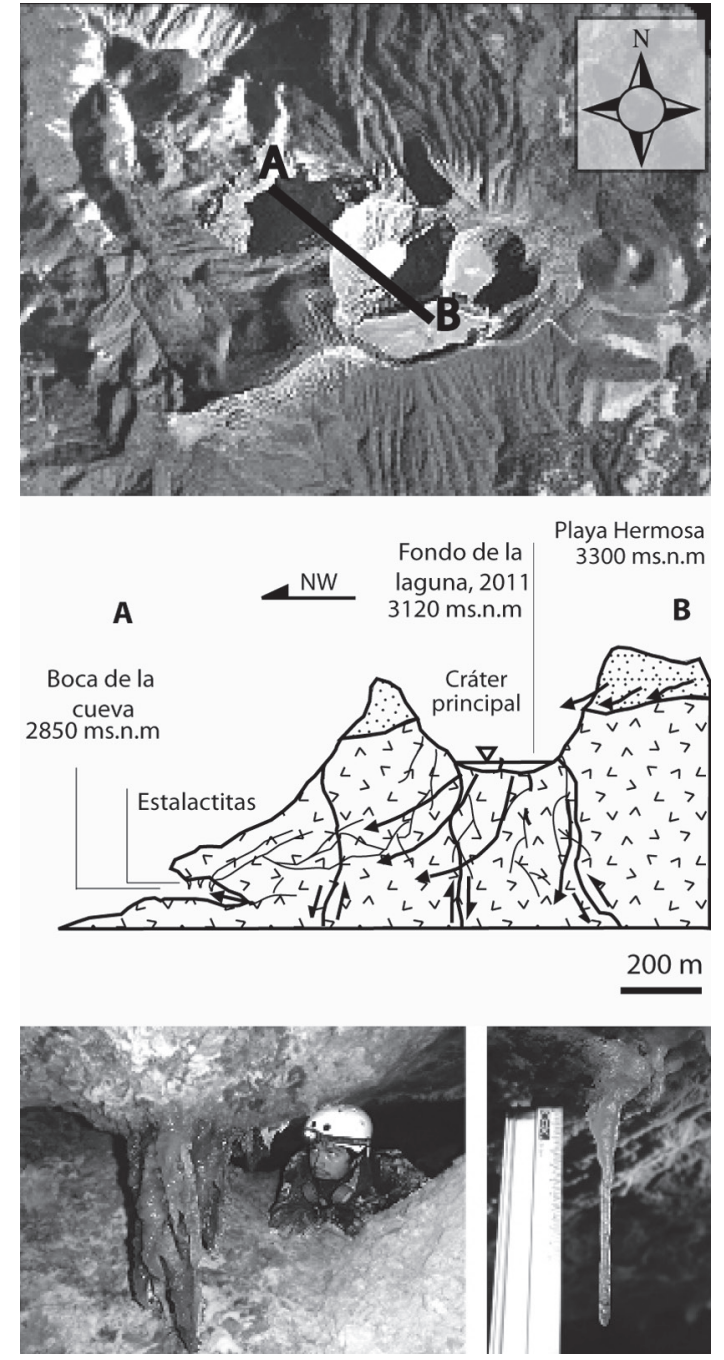

Fig. 9: Perfil A-B, donde se proponen las posibles fracturas en el substrato de la laguna y un desnivel favorable hacia el NW, donde se localiza la cueva. En la parte inferior, se aprecian dos estalactitas, la de la izquierda de aproximadamente $50 \mathrm{~cm}$ y la de la derecha de $15 \mathrm{~cm}$ (Fotografías por A. Ulloa, 2011, izquierda, y V. Carvajal, 2011, derecha).

Los deslizamientos y desprendimientos de rocas que ocurren en los flancos internos del cráter principal, por un lado, más que producir un cambio en el nivel de la laguna, aportan arcillas, materiales finos y de alteración, que podrían estar modificando el color de la laguna inmediatamente después del movimiento del material, retornando a su color anterior o bien a uno distinto, conforme 
los sedimentos se van depositando en el fondo. Por otro lado, los bloques rocosos alteran la condición plástica de los sedimentos, deformándolos y eventualmente produciendo rupturas de las delgadas capas de arcillas, posibilitando de esa manera la filtración de agua de la laguna.

El color, temperatura $\left(16^{\circ}-35^{\circ} \mathrm{C}\right)$ y $\mathrm{pH}(3,0-$ $5,85)$ de la laguna cratérica aparentemente tiene relación con las fumarolas sub-acuáticas, además de los sedimentos en suspensión aportados por los deslizamientos y de algunos organismos que se han adaptado a ese medio.

El drenaje subterráneo inmediato en la laguna cratérica, se debe posiblemente a la contracción y dilatación de planos en las capas delgadas de arcillas que se alternan con capas más gruesas de arenas permeables en la parte basal de la laguna. Los eventos sísmicos pueden jugar un papel importante en las variaciones de los niveles de la laguna, ya que representan la fuerza externa que podrían estar cambiando el comportamiento de las capas de arcillas, fracturándolas y/o produciendo acomodamiento de bloques y sedimentos con generación de rupturas de las capas de arcillas. Además, la existencia de arcillas y sales, que se depositan y precipitan dentro de las fracturas, causan sellamiento temporal, que se puede abrir parcial o totalmente por la acción sísmica y permitir la circulación de fluidos a niveles más profundos. Estas fracturas estarán muy posiblemente conectadas con las cavernas del flanco norte de la cima del Irazú, dando drenajes hacia el río Sucio. Los años en que se ha informado que la laguna ha estado seca son: 1977 , 1979, 1982-83, 1987 y 1990.

La laguna del volcán Irazú presenta registros históricos de niveles máximos de profundidad que rondan los $31 \mathrm{~m}$, no obstante, el nivel promedio máximo de la zona más homogénea en el fondo del cráter es de 17,4 m.

Las causas propuestas para la existencia de la laguna, debe de estar relacionada con la impermeabilización de su fondo con arcillas y sellado de las fracturas, mientras que su rápido drenado después de un tiempo, puede estar condicionado a la contracción de arcillas, disolución de sales e hidrofracturación. Se debe demostrar, sin embargo, de si efectivamente las aguas de la laguna tienen una conexión con las cavidades del sector NW del volcán.

\section{RECOMENDACIONES}

La medición de la profundidad del lago se ha dado de una forma empírica por medio de puntos de referencia los cuales pueden cambiar o desaparecer con el tiempo, se recomienda un sistema de medición semejante al utilizado por Tassi en el 2001 o bien algún tipo de medición con dispositivos de posicionamiento global, de tal manera se pueda tener un dato consistente y se indique si este representa un valor promedio o un valor máximo.

Para poder confirmar algunas de las hipótesis propuestas en este documento, se recomienda realizar un estudio hidrogeológico de la cuenca que se encuentra dentro y en los alrededores del cráter, así como una investigación de las algas y micro-organismos presentes dentro de la laguna. De esta forma, se tendrá una mayor comprensión del origen de las aguas que rellenan el cráter y del destino de las mismas al drenar la laguna. El estudio deberá incluir la geoquímica de los elementos mayores y menores de las aguas de la laguna, del río Sucio, aguas de lluvia y de manantiales, con el objetivo de encontrar una conectividad geoquímica.

Un estudio de isótopos naturales empleando deuterio y $\mathrm{O}^{18}$, en las aguas de la laguna, agua de lluvia, manantiales y el río Sucio, para demostrar la existencia de alguna conexión hidráulica del agua de la laguna y los cuerpos de agua mencionados.

Se recomienda un estudio de subsidencia del fondo de la laguna mediante medidas sistemáticas, con tal de establecer si hay alguna relación entre la constante sedimentación dentro de la laguna y las fuertes variaciones en la profundidad.

\section{AGRADECIMIENTOS}

Se le agradece su valiosa colaboración al geólogo Mauricio Mora Fernández por la obtención de los permisos para el estudio en el Parque Nacional volcán Irazú y el apoyo para la facilitación de 
datos sismológicos. Karina Hernández y personal del Instituto Meteorológico Nacional y Sadí Laporte (Instituto Costarricense de Electricidad) por los datos de precipitación de la estación volcán Irazú. A María Martínez del OVSICORI por brindarnos información del volcán Irazú. Por las facilidades y atenciones en el parque, se les agradece a Rafael Gutiérrez Rojas, Mauricio Gamboa y demás funcionarios del MINAET del Parque Nacional Volcán Irazú. Al grupo Espeleológico Anthros, a Víctor Carvajal por su fotografía, y un agradecimiento especial a Andrés Ulloa Carmiol, por su apoyo técnico en el descenso hasta la laguna. Por el aporte de fotografías históricas, se les agradece a Carlos Goicoechea, Frank Alvarado, Melvin Smart, Federico Gutiérrez, Gerardo Soto, Raúl Mora, Wilson Vindas, Evelyn Cubero, José F. Cervantes, Rafael Barquero y Percy Denyer. También se le agradece a Julie A. Herrick, José Viramonte y Walter Hernández por su valiosa colaboración en la revisión del presente artículo.

\section{REFERENCIAS}

ALVARADO, G., 1989: Los volcanes de Costa Rica.- 175 págs. EUNED, San José.

ALVARADO, G., 2000: Volcanes de Costa Rica: Geología, historia y riqueza natural.- 269 págs. EUNED, San José.

BARQUERO, J., 1998: Volcán Irazú, Costa Rica.50 págs. Imp. Lihssa, San José.

BARQUERO, R., SOTO, G. \& LESAGE, P., 1992: Informe vulcanológico enero 1991 - mayo 1992.- 65 págs. Oficina de Vulcanología y Sismología, ICE; Univ. Sanboya, Francia; Sección de Sismología y Vulcanología, Red Sismológica Nacional UCR-ICE [Inf. interno].

BARQUERO, R. MORA, M., MADRIGAL, L., VARGAS, I., ARIAS, F. \& SOTO, G.., 1995: Resumen anual de sismos sentidos y actividad volcánica en Costa Rica.- 19 págs.
Oficina de Vulcanología y Sismología, ICE, Red Sismológica Nacional UCR-ICE [Inf. interno].

FERNÁNDEZ, E. \& DUARTE, E., 2010a: Fondo del lago cratérico del Volcán Irazú reducido a menos de $1 \mathrm{~m}$ de profundidad.- 2 págs. OVSICORI-UNA, Heredia [Inf. Interno].

FERNÁNDEZ, E. \& DUARTE, E., 2010b: Colonización vegetal del fondo del lago seco del V. Irazú, 22 de Julio del 2010.- 5 págs. OVSICORI-UNA, Heredia [Inf. Interno].

KRUSHENSKY, R.D., 1972: Geology of the Istarú Quadrangle, Costa Rica.- 46 págs. U.S. Geol. Surv.1358.

MCCLELLAND, L., SINKIN, T., SUMMERS, M., NIELSEN, E. \& STEIN, T.C. (eds.) 1989: Global Volcanism 1975-1985.- 465 págs. Prentice-Hall, Inc., New Yersey.

MORA, M., 1997: Informe de la actividad de los volcanes Poás e Irazú 1994-1996.- 64 págs. Sección de Sismología, Vulcanología y Exploración Geofísica, Univ. Costa Rica [Inf. interno].

MORA, R., 2003: Informe anual de la actividad de la Cordillera Volcánica Central, 2002, Costa Rica.- 50 págs. Red Sismológica Nacional UCR-ICE [Inf. Interno].

MORA, R., 2005: Informe de la actividad de la Cordillera Volcánica Central, Enero 2003-Junio 2004.- 50 págs. Red Sismológica Nacional UCR-ICE [Inf. Interno].

OVSICORI, 1979: Volcán Irazú.-- Bol de Vulcano. 3: 4.

OVSICORI, 1980: Volcán Irazú. Estado de los volcanes.- Bol de Vulcanol. 7: 6; 8: 8-9; 9: 11.

OVSICORI, 1981: Volcán Irazú. Estado de los volcanes.- Bol de Vulcanología, 10: 7; 11: 4. 
OVSICORI, 1983: Volcán Irazú. Estado de los volcanes.- Bol de Vulcanología, 13: 4.

OVSICORI, 1986: Volcán Irazú. Estado de los volcanes.- Bol de Vulcanología, 17: 5.

PANIAGUA, S. \& SOTO, G., 1986: Reconocimiento de los riesgos volcánicos potenciales de la Cordillera Central de C.R, América Central.- Ciencia y Tecnología, 10(2): 49-72.

TASSI, F., VASELLI, O., FERNÁNDEZ, E., DUARTE, E., MARTINEZ, M., DELGADO, A. \& BERGAMASCHI, F., 2009: Morphological and geochemical features of crater lakes in Costa Rica: an overview.- J. Limnol. 68(2): 1-13.
TAZIEFF, H., 1972: Los volcanes y la deriva de los continentes.- 127 págs. Ed. Labor S.A, Barcelona.

VAN DER LATT, R., 2009: Descenso de nivel de la laguna cratérica del volcán Irazú, Costa Rica, 2003-2009.- 19 págs. OVSICORIUNA, Heredia [Inf. Interno].

VENZKE, E., WUNDERMAN, R. W., MCCLELLAND, L., SIMKIN, T., LUHR, J. F., SIEBERT, L., MAYBERRY, G. \& SENNERT, S. (eds.): 2002-. Global volcanism 1968 to the Present.- Smithsonian Inst. Global Volcanism Program Digital Information Series. GVP-4.- http://www. volcano.si.edu/reports/. [Consulta: 24 de diciembre del 2012]. 
